Correction

\title{
Correction: Xia, C.; et al. Unified Strength Model of Asphalt Mixture under Various Loading Modes. Materials 2019, 12, 889
}

\section{Chengdong Xia ${ }^{1}\left(\right.$, Songtao $\mathrm{Lv}^{1, *}{ }^{1}$, Lingyun You ${ }^{2}\left(\mathbb{D}\right.$, Dong Chen ${ }^{1,3}$, Yipeng $\mathrm{Li}^{1}$} and Jianlong Zheng ${ }^{1}$

1 National Engineering Laboratory of Highway Maintenance Technology, Changsha University of Science \& Technology, Changsha 410004, China; xiachengdong@stu.csust.edu.cn (C.X.); 15878778765@163.com (D.C.); lypcsust@163.com (Y.L.); zj1@csust.edu.cn (J.Z.)

2 Department of Civil and Environmental Engineering, Michigan Technological University, 1400 Townsend Drive, Houghton, MI 49931, USA; liyou@mtu.edu

3 Guangxi Communications Design Group Co., Ltd., Nanning 530000, China

* Correspondence: 1st@csust.edu.cn

Received: 16 November 2020; Accepted: 25 November 2020; Published: 27 November 2020

\section{Change in Tables}

The authors wish to make the following correction to this paper [1]. Due to mislabeling, replace:

Table 6. Indirect tensile strength test of asphalt mixture.

\begin{tabular}{|c|c|c|c|c|c|c|}
\hline Number & $\begin{array}{l}\text { Loading Rate } v \\
(\mathrm{MPa} / \mathrm{s})\end{array}$ & $\begin{array}{c}\text { Section Area of } \\
\text { Specimen } A \\
\left(\mathrm{~mm}^{2}\right)\end{array}$ & $\begin{array}{l}\text { Failure } \\
\text { Loading } F \\
\quad(\mathbf{k N})\end{array}$ & $\begin{array}{c}\text { Strength } \\
R_{\mathrm{D}} \text { (MPa) }\end{array}$ & $\begin{array}{l}\text { Average Value of } \\
\text { Strength } R_{\mathrm{D}} \\
\text { (MPa) }\end{array}$ & $\begin{array}{l}\text { Coefficient of } \\
\text { Variation }\end{array}$ \\
\hline 1 & \multirow{3}{*}{5} & 58.5 & 28.948 & 3.111 & \multirow{3}{*}{3.258} & \multirow{3}{*}{0.044} \\
\hline 2 & & 60.4 & 33.164 & 3.452 & & \\
\hline 3 & & 60.5 & 30.900 & 3.211 & & \\
\hline 4 & \multirow{3}{*}{10} & 60.1 & 34.997 & 3.661 & \multirow{3}{*}{3.704} & \multirow{3}{*}{0.022} \\
\hline 5 & & 58.9 & 35.778 & 3.819 & & \\
\hline 6 & & 59.1 & 34.142 & 3.632 & & \\
\hline 7 & \multirow{3}{*}{20} & 59.3 & 40.709 & 4.316 & \multirow{3}{*}{4.41} & \multirow{3}{*}{0.041} \\
\hline 8 & & 59.6 & 40.299 & 4.251 & & \\
\hline 9 & & 58.8 & 43.611 & 4.663 & & \\
\hline 10 & \multirow{3}{*}{30} & 59.2 & 46.808 & 4.971 & \multirow{3}{*}{4.837} & \multirow{3}{*}{0.026} \\
\hline 11 & & 59.7 & 44.307 & 4.666 & & \\
\hline 12 & & 59.6 & 46.205 & 4.874 & & \\
\hline 13 & \multirow{3}{*}{40} & 59 & 47.542 & 5.066 & \multirow{3}{*}{5.185} & \multirow{3}{*}{0.020} \\
\hline 14 & & 59.8 & 50.621 & 5.322 & & \\
\hline 15 & & 60.1 & 49.393 & 5.167 & & \\
\hline 16 & \multirow{3}{*}{50} & 59.7 & 53.129 & 5.595 & \multirow{3}{*}{5.487} & \multirow{3}{*}{0.037} \\
\hline 17 & & 61.2 & 55.106 & 5.661 & & \\
\hline 18 & & 59.6 & 49.343 & 5.205 & & \\
\hline 19 & \multirow{3}{*}{60} & 60.5 & 55.592 & 5.777 & \multirow{3}{*}{5.658} & \multirow{3}{*}{0.035} \\
\hline 20 & & 61.4 & 56.810 & 5.817 & & \\
\hline 21 & & 60.1 & 51.430 & 5.38 & & \\
\hline 22 & \multirow{3}{*}{70} & 61.7 & 57.814 & 5.891 & \multirow{3}{*}{5.784} & \multirow{3}{*}{0.029} \\
\hline 23 & & 60.3 & 53.241 & 5.551 & & \\
\hline 24 & & 60.8 & 57.154 & 5.91 & & \\
\hline
\end{tabular}


with

Table 6. Indirect tensile strength test of asphalt mixture.

\begin{tabular}{|c|c|c|c|c|c|c|}
\hline Number & $\begin{array}{l}\text { Loading Rate } v \\
\quad(\mathrm{MPa} / \mathrm{s})\end{array}$ & $\begin{array}{c}\text { Height of } \\
\text { Specimen } h(\mathrm{~mm})\end{array}$ & $\begin{array}{l}\text { Failure } \\
\text { Loading } F \\
\quad(k N)\end{array}$ & $\begin{array}{c}\text { Strength } \\
R_{\mathrm{T}}(\mathrm{MPa})\end{array}$ & $\begin{array}{l}\text { Average Value of } \\
\text { Strength } R_{\mathrm{T}} \\
(\mathrm{MPa})\end{array}$ & $\begin{array}{l}\text { Coefficient of } \\
\text { Variation }\end{array}$ \\
\hline 1 & \multirow{3}{*}{5} & 58.5 & 28.948 & 3.111 & \multirow{3}{*}{3.258} & \multirow{3}{*}{0.044} \\
\hline 2 & & 60.4 & 33.164 & 3.452 & & \\
\hline 3 & & 60.5 & 30.900 & 3.211 & & \\
\hline 4 & \multirow{3}{*}{10} & 60.1 & 34.997 & 3.661 & \multirow{3}{*}{3.704} & \multirow{3}{*}{0.022} \\
\hline 5 & & 58.9 & 35.778 & 3.819 & & \\
\hline 6 & & 59.1 & 34.142 & 3.632 & & \\
\hline 7 & \multirow{3}{*}{20} & 59.3 & 40.709 & 4.316 & \multirow{3}{*}{4.41} & \multirow{3}{*}{0.041} \\
\hline 8 & & 59.6 & 40.299 & 4.251 & & \\
\hline 9 & & 58.8 & 43.611 & 4.663 & & \\
\hline 10 & \multirow{3}{*}{30} & 59.2 & 46.808 & 4.971 & \multirow{3}{*}{4.837} & \multirow{3}{*}{0.026} \\
\hline 11 & & 59.7 & 44.307 & 4.666 & & \\
\hline 12 & & 59.6 & 46.205 & 4.874 & & \\
\hline 13 & \multirow{3}{*}{40} & 59 & 47.542 & 5.066 & \multirow{3}{*}{5.185} & \multirow{3}{*}{0.020} \\
\hline 14 & & 59.8 & 50.621 & 5.322 & & \\
\hline 15 & & 60.1 & 49.393 & 5.167 & & \\
\hline 16 & \multirow{3}{*}{50} & 59.7 & 53.129 & 5.595 & \multirow{3}{*}{5.487} & \multirow{3}{*}{0.037} \\
\hline 17 & & 61.2 & 55.106 & 5.661 & & \\
\hline 18 & & 59.6 & 49.343 & 5.205 & & \\
\hline 19 & \multirow{3}{*}{60} & 60.5 & 55.592 & 5.777 & \multirow{3}{*}{5.658} & \multirow{3}{*}{0.035} \\
\hline 20 & & 61.4 & 56.810 & 5.817 & & \\
\hline 21 & & 60.1 & 51.430 & 5.38 & & \\
\hline 22 & \multirow{3}{*}{70} & 61.7 & 57.814 & 5.891 & \multirow{3}{*}{5.784} & \multirow{3}{*}{0.029} \\
\hline 23 & & 60.3 & 53.241 & 5.551 & & \\
\hline 24 & & 60.8 & 57.154 & 5.91 & & \\
\hline
\end{tabular}

The authors wish to make the following correction to this paper [1]. Due to mislabeling, replace: 
Table 7. Test results of unconfined compressive strength of asphalt mixture.

\begin{tabular}{|c|c|c|c|c|c|c|}
\hline Number & $\begin{array}{c}\text { Loading Rate } v \\
(\mathrm{MPa} / \mathrm{s})\end{array}$ & $\begin{array}{l}\text { Section Area of } \\
\text { Specimen } A \\
\quad\left(\mathrm{~mm}^{2}\right)\end{array}$ & $\begin{array}{l}\text { Failure } \\
\text { Loading } F \\
\text { (kN) }\end{array}$ & $\begin{array}{l}\text { Strength } \\
R_{\mathrm{D}}(\mathrm{MPa})\end{array}$ & $\begin{array}{l}\text { Average Value of } \\
\text { Strength } R_{\mathrm{D}} \\
\text { (MPa) }\end{array}$ & $\begin{array}{l}\text { Coefficient of } \\
\text { Variation }\end{array}$ \\
\hline 1 & \multirow{3}{*}{0.02} & 34.862 & 34.862 & 4.441 & \multirow{3}{*}{4.134} & \multirow{3}{*}{0.057} \\
\hline 2 & & 32.169 & 32.169 & 4.098 & & \\
\hline 3 & & 30.325 & 30.325 & 3.863 & & \\
\hline 4 & \multirow{3}{*}{0.05} & 38.473 & 38.473 & 4.901 & \multirow{3}{*}{5.062} & \multirow{3}{*}{0.039} \\
\hline 5 & & 38.795 & 38.795 & 4.942 & & \\
\hline 6 & & 41.943 & 41.943 & 5.343 & & \\
\hline 7 & \multirow{3}{*}{0.1} & 47.249 & 47.249 & 6.019 & \multirow{3}{*}{5.901} & \multirow{3}{*}{0.025} \\
\hline 8 & & 46.998 & 46.998 & 5.987 & & \\
\hline 9 & & 44.721 & 44.721 & 5.697 & & \\
\hline 10 & \multirow{3}{*}{0.5} & 63.773 & 63.773 & 8.124 & \multirow{3}{*}{8.421} & \multirow{3}{*}{0.025} \\
\hline 11 & & 66.851 & 66.851 & 8.516 & & \\
\hline 12 & & 67.691 & 67.691 & 8.623 & & \\
\hline 13 & \multirow{3}{*}{1} & 78.429 & 78.429 & 9.991 & \multirow{3}{*}{9.816} & \multirow{3}{*}{0.013} \\
\hline 14 & & 76.255 & 76.255 & 9.714 & & \\
\hline 15 & & 76.483 & 76.483 & 9.743 & & \\
\hline 16 & \multirow{3}{*}{2} & 87.064 & 87.064 & 11.091 & \multirow{3}{*}{11.441} & \multirow{3}{*}{0.022} \\
\hline 17 & & 90.636 & 90.636 & 11.546 & & \\
\hline 18 & & 91.735 & 91.735 & 11.686 & & \\
\hline
\end{tabular}

with

Table 7. Test results of unconfined compressive strength of asphalt mixture.

\begin{tabular}{|c|c|c|c|c|c|c|}
\hline Number & $\begin{array}{l}\text { Loading Rate } v \\
(\mathrm{MPa} / \mathrm{s})\end{array}$ & $\begin{array}{c}\text { Section Area of } \\
\text { Specimen } A \\
\left(\mathrm{~mm}^{2}\right)\end{array}$ & $\begin{array}{l}\text { Failure } \\
\text { Loading } F \\
(\mathrm{kN})\end{array}$ & $\begin{array}{l}\text { Strength } \\
R_{\mathrm{C}}(\mathrm{MPa})\end{array}$ & $\begin{array}{l}\text { Average Value of } \\
\text { Strength } R_{\mathrm{C}} \\
\text { (MPa) }\end{array}$ & $\begin{array}{l}\text { Coefficient of } \\
\text { Variation }\end{array}$ \\
\hline 1 & \multirow{3}{*}{0.02} & 7850.03 & 34.862 & 4.441 & \multirow{3}{*}{4.134} & \multirow{3}{*}{0.057} \\
\hline 2 & & 7849.93 & 32.169 & 4.098 & & \\
\hline 3 & & 7850.12 & 30.325 & 3.863 & & \\
\hline 4 & \multirow{3}{*}{0.05} & 7850.03 & 38.473 & 4.901 & \multirow{3}{*}{5.062} & \multirow{3}{*}{0.039} \\
\hline 5 & & 7850.06 & 38.795 & 4.942 & & \\
\hline 6 & & 7850.08 & 41.943 & 5.343 & & \\
\hline 7 & \multirow{3}{*}{0.1} & 7849.98 & 47.249 & 6.019 & \multirow{3}{*}{5.901} & \multirow{3}{*}{0.025} \\
\hline 8 & & 7850.01 & 46.998 & 5.987 & & \\
\hline 9 & & 7849.92 & 44.721 & 5.697 & & \\
\hline 10 & \multirow{3}{*}{0.5} & 7849.95 & 63.773 & 8.124 & \multirow{3}{*}{8.421} & \multirow{3}{*}{0.025} \\
\hline 11 & & 7850.05 & 66.851 & 8.516 & & \\
\hline 12 & & 7850.05 & 67.691 & 8.623 & & \\
\hline 13 & \multirow{3}{*}{1} & 7849.96 & 78.429 & 9.991 & \multirow{3}{*}{9.816} & \multirow{3}{*}{0.013} \\
\hline 14 & & 7850.01 & 76.255 & 9.714 & & \\
\hline 15 & & 7850.05 & 76.483 & 9.743 & & \\
\hline 16 & \multirow{3}{*}{2} & 7849.97 & 87.064 & 11.091 & \multirow{3}{*}{11.441} & \multirow{3}{*}{0.022} \\
\hline 17 & & 7849.99 & 90.636 & 11.546 & & \\
\hline 18 & & 7849.99 & 91.735 & 11.686 & & \\
\hline
\end{tabular}

The authors would like to apologize for any inconvenience caused to the readers by these changes. 


\section{Reference}

1. Xia, C.; Lv, S.; You, L.; Chen, D.; Li, Y.; Zheng, J. Unified strength model of asphalt mixture under various loading modes. Materials 2019, 12, 889. [CrossRef] [PubMed]

Publisher's Note: MDPI stays neutral with regard to jurisdictional claims in published maps and institutional affiliations.

(C) 2020 by the authors. Licensee MDPI, Basel, Switzerland. This article is an open access article distributed under the terms and conditions of the Creative Commons Attribution (CC BY) license (http://creativecommons.org/licenses/by/4.0/). 